\title{
Possible demonstration of ionization cooling using absorbers in a solenoidal field
}

R.C. Fernow, J.C. Gallardo, H.G. Kirk, T. Kycia, Y.Y. Lee, L. Littenberg, R.B. Palmer, V. Polychronakos \& I. Stumer Brookhaven National Laboratory, Upton, NY 11973

\author{
D.V. Neuffer \\ CEBAF, Newport News, VA 23606 \\ D. R. Winn \\ Fairfield University, Fairfield, CT 06430 \\ R. Thun \& R. Ball \\ University of Michigan, Ann Arbor, MI 48109 \\ M. Marx \& Y. Torun \\ SUNY at Stony Brook, Stony Brook, NY 11794 \\ M. Zeller \\ Yale University, New Haven, CT 06511
}

\begin{abstract}
Ionization cooling may play an important role in reducing the phase space volume of muons for a future muon-muon collider. We describe a possible experiment to demonstrate transverse emittance cooling using a muon beam at the AGS at Brookhaven National Laboratory. The experiment uses device dimensions and parameters and beam conditions similar to what is expected in an actual muon-muon collider.
\end{abstract}




\section{Introduction}

There has been considerable recent interest in the development of high energy colliders using muon beams[1]. Most of the proposed designs require a high intensity pion beam resulting from protons interacting in a stationary target. The muons are collected from the decaying pions. However, the resulting muon beam typically has much too large an emittance to give high luminosity in a collider.

It is possible to reduce the transverse emittance of the muon beam through the process of ionization cooling[2]. Energy loss in a material reduces both the transverse and longitudinal components of the momentum. Multiple scattering in the material is a competing process that effectively heats the beam, resulting in an increase of the emittance. Subsequent acceleration cavities restore only the longitudinal component, thus leading to a reduction of the transverse emittance of the muon beam.

Transverse ionization cooling, although discussed by Skrinsky and others[3] in the 70's has, as far as we know, never been observed. Its demonstration is important because such cooling appears to allow the design of high luminosity muon-muon colliders that may provide the best possibility for physics at $\mathrm{CM}$ energies above $1 \mathrm{TeV}$.

We are considering the possibility of developing a prototype cooling system and testing its performance in a muon beam at the AGS at Brookhaven National Laboratory. A muon momentum of $210 \mathrm{MeV} / \mathrm{c}$ would be ideal for the experiment.

\section{Ionization cooling}

In order to generate sufficient muons it is necessary to capture a very large fraction of the pions generated at the target. These pions, and the muons into which they decay, are then necessarily very diffuse (i.e. they have a very large emittance). Since luminosity is inversely proportional to the final beam cross section, the beam must be small. Therefore, some form of cooling to lower the emittance is essential.

The large mass of the muon compared to that of the electron prevents cooling by radiation damping, while the short lifetime of the muon prevents conventional stochastic or electron cooling. Fortunately, the process of ionization cooling, which because of their long interaction length is possible only for muons, can be used. In this process the muon loses transverse and longitudinal momentum by $\mathrm{dE} / \mathrm{dx}$ in a material, such as lithium or beryllium, and then has the longitudinal momentum (but not the transverse momentum) restored in a subsequent RF cavity. 
The combined effect is to reduce the beam divergence and thus the emittance of the beam. The process is complicated by the simultaneous presence of multiple scattering in the material, which acts as a source of "heat" and increases the emittance. The cooling effect can dominate for low $\mathrm{Z}$ materials in the presence of strong focussing fields. One solution being considered for the collider is to use rods of lithium or beryllium inside a solenoid magnet. The rod provides the energy loss, while the large aperture solenoid provides the required focussing.

While the physics of the cooling process is rather well understood, the technology of a system that achieves it is not. As we mentioned in the Introduction, such cooling has never been convincingly observed. If a muon collider based on ionization cooling is to be proposed, then we believe that it is essential that such cooling must first be demonstrated.

The normalized transverse emittance $\varepsilon_{\mathrm{n}}$ can be reduced by ionization energy loss $\mathrm{dE} / \mathrm{dz}$ in a material at the rate[4]

$$
\frac{d \epsilon_{n}}{d z}=-\frac{1}{\beta^{2}} \frac{d E}{d z} \frac{\epsilon_{n}}{E}
$$

where $E=\gamma m_{p} c^{2}$ is the total energy of the muon. At a momentum corresponding to minimum ionization, $\mathrm{dE} / \mathrm{dz}$ is $0.88 \mathrm{MeV} / \mathrm{cm}$ for lithium and $2.95 \mathrm{MeV} / \mathrm{cm}$ for beryllium. In practical units we can write Eq. 1 as

$$
\frac{d \epsilon_{n}}{d z}\left[\frac{m m-m r a d}{c m}\right]=-\frac{1}{\beta^{2}} \frac{d E}{d z}\left[\frac{M e V}{C m}\right] \frac{\epsilon_{n}[m m-m r a d]}{E[M e V]}
$$

The rod also introduces heating of the normalized transverse emittance due to multiple scattering at a rate[4]

$$
\frac{d \epsilon_{n}}{d z}=\frac{\beta_{\perp}}{2 \beta^{3}} \frac{E_{s}^{2}}{E m_{\mu} C^{2}} \frac{1}{L_{r}}
$$

where $\beta_{\perp}$ is betatron focusing parameter, $\beta$ is the relativistic velocity of the muon, $E_{\mathrm{s}} \approx 14 \mathrm{MeV}$ is the characteristic energy for the projected scattering angle from multiple scattering theory, $\mathrm{m}_{\mu} \mathrm{c}^{2}$ is the rest energy of the muon $(105.6 \mathrm{MeV})$, and $L_{r}$ is the radiation length of the material $(155 \mathrm{~cm}$ for lithium and $35.3 \mathrm{~cm}$ for beryllium ). 
In practical units Eq. 3 can be written as

$$
\frac{d \epsilon_{n}[\mathrm{~mm}-m \mathrm{mad}]}{d z[\mathrm{Cm}]}=\frac{60 \beta_{\perp}[\mathrm{cm}]}{\beta^{3} E[\mathrm{MeV}]}
$$

for lithium. The minimum achievable emittance, which occurs when the cooling rate equals the heating rate, is given by

$$
\min \epsilon_{N}=\frac{\beta_{\perp} E_{S}^{2}}{2 \beta m_{\mu} C^{2} L_{R} \frac{d E}{d z}}
$$

In practical units

$$
\min \epsilon_{N}[m m-m r a d]=\frac{9280}{\beta L_{R}[c m] \frac{d E}{d z}\left[\frac{M e V}{C m}\right]} \beta_{\perp}[\mathrm{cm}]
$$

For a relativistic particle the coefficient of $\beta_{\perp}$ is 68.0 for lithium and 89.1 for beryllium. The betatron focusing parameter for a solenoid is given by

$$
\beta_{\perp}=\frac{2 p_{z}}{e B}
$$

or in practical units

$$
\beta_{\perp}[\mathrm{Cm}]=0.667 \frac{p_{Z}[\mathrm{MeV} / \mathrm{C}]}{B[\mathrm{~T}]}
$$

A reduction in transverse emittance is accompanied by a corresponding increase in longitudinal emittance due to straggling. This introduces a spread in the mean energy loss in the material given by[4]

$$
\frac{d\left(\sigma_{E}^{2}\right)}{d z}=4 \pi\left(r_{\theta} m_{\theta} C^{2}\right)^{2} n_{e} \gamma^{2}\left(1-1 / 2 \beta^{2}\right)
$$

where $r_{e}$ is the classical radius of the electron, $m_{e} c^{2}$ is the rest energy of the electron, $\gamma$ and $\beta$ are the usual relativistic factors, and $n_{e}=Z N_{A} \rho / A$ is the number of electrons per unit volume of absorber material, where $Z, A, \rho$ are the atomic number, atomic weight and density of the material. 


\section{Outline of the proposed experiment}

We propose building a prototype cooling cell with properties as close as possible to those required in a muon collider. We would measure the performance of the device and compare it with predictions from the Monte Carlo programs we are using to design the collider. Since the cooling section may represent a large fraction of the total cost of a muon collider, it is essential that the design Monte Carlo accurately model the achievable cooling. We also need to check that muon losses in the focusing fields and absorbing material do not exceed the predicted amounts.

The proposed baseline experiment would use a cylindrical piece of lithium, $12 \mathrm{~cm}$ in radius and $60 \mathrm{~cm}$ in length as the cooling rod. The solenoid with a maximum central field strength of $7 \mathrm{~T}$ would focus the muons in the interior of the rod. The absorber material would be replaced at some point with different lengths of absorber and with $\mathrm{Be}$ or $\mathrm{LiH}$ absorbers in order to further check the accuracy of the Monte Carlo predictions. Beryllium would produce more efficient cooling than lithium for some regions of the muon parameters.

In order to simulate the conditions in the muon collider as closely as possible, the momentum of the incident muon should be around $210 \mathrm{MeV} / \mathrm{c}$. For a $0.6 \mathrm{~m}$ lithium rod the final momentum would be about $140 \mathrm{MeV}$. The focusing $\beta_{\perp}$ in the rod should be as small as possible, since Eq. 5 shows that the minimum emittance that can be reached is proportional to $\beta_{\perp}$.

The layout of the proposed experiment is shown in Fig. 1. A large, diffuse muon beam passes through a hadron absorber and enters an approximately $6 \mathrm{~m}$ long experimental area. The dipole D1 together with the position measuring detectors P1-5 are used to measure the momentum of each incoming track. A fraction of the beam will be captured by the solenoid magnet $S 1$ and focused into an absorber. The momentum of the muons leaving the solenoid is measured using dipole magnet D2 and detectors P6-10. Trigger counters would tag tracks entering the absorber. A time of flight system would be necessary to identify pion and electron background in the muon beam. The dipole D1 would have to be adjusted to examine a series of different central values for the incident momentum. The momentum spread could be determined by software selection of the tracks used in the analysis. 


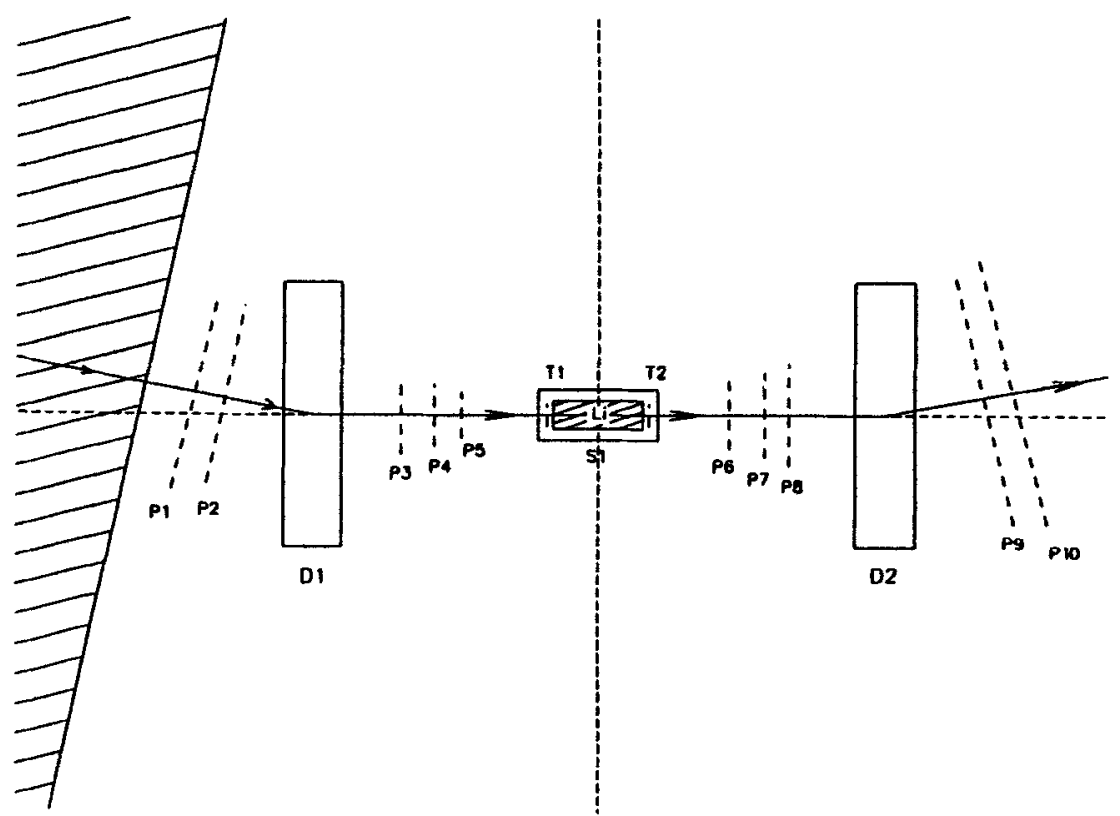

Fig.1 . Layout of the proposed experiment. D1,2 are dipole magnets, $\mathrm{S} 1$ is a solenoid magnet, $\mathrm{P} 1-10$ are position measuring detectors.

In the following we consider an example case with an initial normalized emittance of $4000 \mathrm{~mm}-\mathrm{mrad}$ and a $7 \mathrm{~T}$ solenoid field. Some properties of the magnets are listed in Table 1. The dipole width and length are appropriate to existing 72D18 magnets at the AGS. We assume a bend angle in the dipoles of $0.2 \mathrm{rad}$.

\begin{tabular}{||l|c|c|c|c|c||}
\hline \multicolumn{7}{|c|}{ Table 1 Magnet summary } \\
\hline Magnet & length & width & gap & radius & B $_{\mathbf{o}}$ \\
\hline & {$[\mathrm{cm}]$} & {$[\mathrm{cm}]$} & {$[\mathrm{cm}]$} & {$[\mathrm{cm}]$} & {$[\mathrm{T}]$} \\
\hline D1 & 46 & 183 & 109 & - & 0.30 \\
\hline S1 & 80 & - & - & 15 & 7.00 \\
\hline D2 & 46 & 183 & 148 & - & 0.20 \\
\hline
\end{tabular}


a $1 \sigma$ radius of about $3 \mathrm{~cm}$ inside the lithium. PARMELA results for the change in transverse normalized emitance as a function of axial distance $\mathrm{z}$ are shown in Fig. 2 and summarized in Table 2.

\begin{tabular}{||l|l|l|l||}
\hline \multicolumn{4}{|c||}{ Table 2 Transverse cooling experiment } \\
\hline & $\mathrm{IN}$ & OUT & \\
\hline $\mathrm{T}$ & 130 & 70 & $\mathrm{MeV}$ \\
\hline $\mathrm{p}$ & 211 & 140 & $\mathrm{MeV} / \mathrm{c}$ \\
\hline$\beta$ & 0.894 & 0.798 & \\
\hline$\gamma$ & 2.23 & 1.66 & \\
\hline$\varepsilon_{\mathrm{N}}$ & 4000 & 3100 & $\mathrm{~mm}-\mathrm{mrad}$ \\
\hline$\varepsilon_{\mathrm{G}}$ & 2006 & 2340 & $\mathrm{~mm}-\mathrm{mrad}$ \\
\hline$\beta_{\perp}(\mathrm{sol})$ & 20.1 & 13.3 & $\mathrm{~cm}$ \\
\hline$\sigma_{\mathrm{X}}$ & 20 & 18 & $\mathrm{~mm}$ \\
\hline$\sigma_{\mathrm{X}}$ & 100 & 133 & $\mathrm{mrad}$ \\
\hline \hline
\end{tabular}

Under the conditions listed in Table 2, the cooling rate is always larger than the heating rate and the normalized emittance of the beam leaving the rod is smaller than the normalized emittance entering it. However, the geometric emittance increases after traversing the rod. In an actual muon collider this stage must be followed by a reacceleration section that restores the starting momentum in order to get cooling of the geometric emittance.

One of the design requirements for the experiment is the ability to measure individual muon tracks. This will permit software "control" of the effective cooling rate. Software selection of initial beam tracks will allow us to reconstruct final beam emittance as a function of initial beam emittance. 


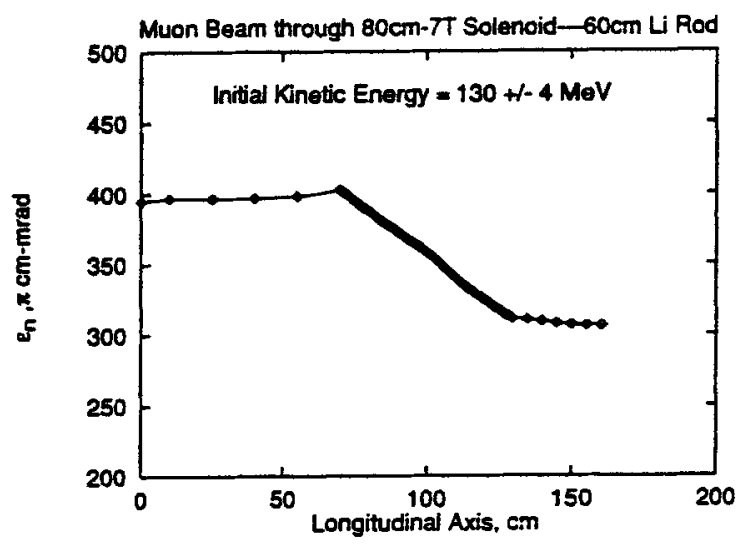

Fig. 2 PARMELA simulation of the normalized transverse emittance as a function of axial distance. The incident muon beam has a normalized emittance of $4000 \mathrm{~mm}$-mrad.

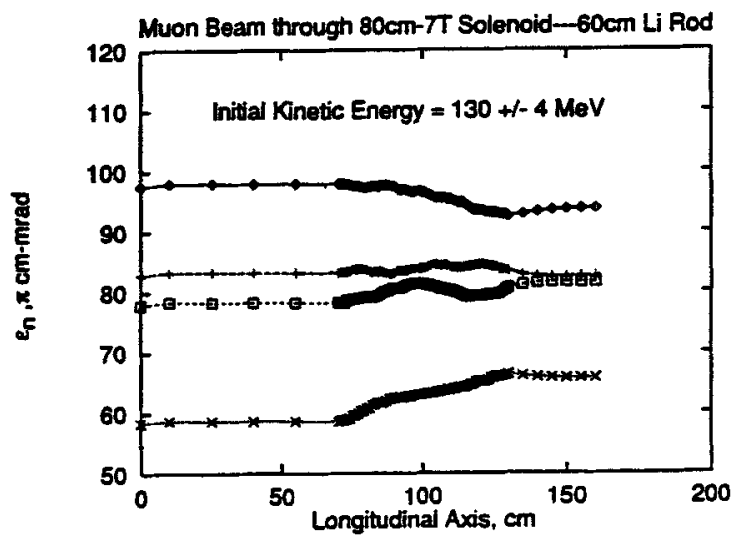

Fig. 3 PARMELA simulation of the change in normalized transverse emittance as a function of axial distance for smaller initial values of the emittance. 
The experiment can be reanalyzed for smaller initial transverse emittances. As the initial emittances decrease, the cooling in the lithium becomes less effective relative to the heating and the net decrease in normalized emitance also drops. Fig. 3 shows that this cooling configuration should still produce net cooling until the initial emittance reaches about $820 \mathrm{~mm}$-mrad. These measurements are important since they demonstrate the minimum emittance achievable for a given field strength and absorber length.

The region of the incident beam phase space for an initial emittance of $4000 \mathrm{~mm}$ mrad that leads to cooling is shown in Fig. 4. The tilted ellipse represents a converging beam. Incident beams with smaller initial emittances are subsets of the data shown in the figure. The basic idea would be to use an incident beam phase space that completely covers the cooling region in Fig. 4. Then, since individual trajectories are measured, we would select by software those trajectories that lie in the cooling region. These trajectories should produce a known amount of normalized emittance reduction, as indicated in Fig. 2 and Table 2. Then subsets of these trajectories could be examined and the net cooling compared with the predictions shown in Fig. 3. Trajectories in the core area of Fig. 4 could also be examined to confirm that no cooling occurs.

The experimental test of cooling would require accurately measuring the position, angle, and momentum of individual muon tracks before and after the rod in order to measure the initial and final emittances. The positions have to be measured at 10 locations. Since the chambers are likely to be in the strong fringe field of the solenoid, a good field map will be required. We need at least three $x$ and $y$ measuring planes on each side of the rod to measure the curvature of the track. The six chambers closest to the rod require measurements with moderate resolution $(\approx 300 \mu \mathrm{m})$. 


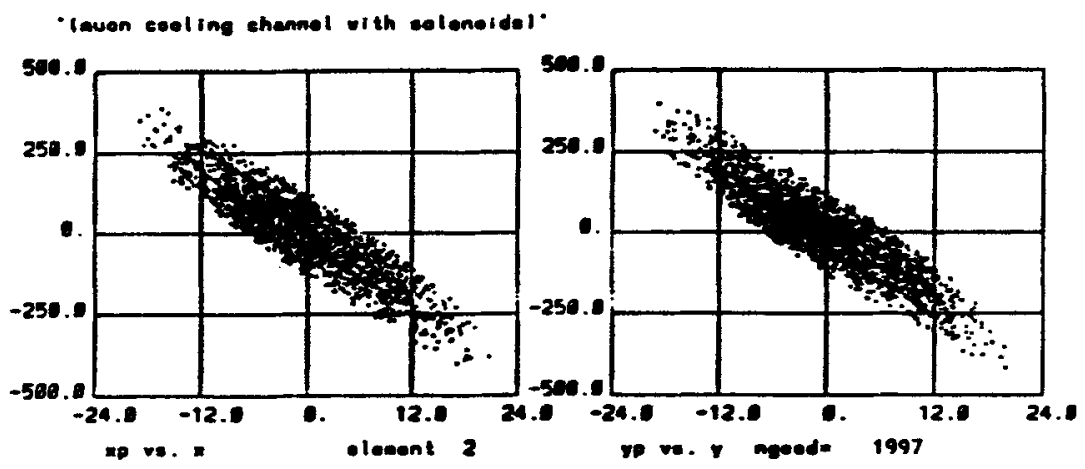

Fig. 4 PARMELA simulation of the $1 \sigma$ geometric incident muon beam phase space that leads to the normalized emittance cooling shown in Fig. 2. (a) $x$ vs $x^{\prime}$; (b) y vs $y^{\prime}$.

The transverse cooling experiments require measuring the normalized muon beam emittance before and after the rod. The normalized emittance at a beam waist is given by

$$
\epsilon_{N}=\frac{\bar{p}}{m_{\mu} c} \sigma_{X} \sigma_{\theta}
$$

where $\sigma_{\mathrm{x}}$ and $\sigma_{\theta}$ are the values for the entrance or the exit to the rod, $\mathrm{p}$ is the mean momentum for the distribution and $\mathrm{m}_{\mathrm{h}}$ is the mass of the muon. 
The fractional error on the measurement of $\varepsilon_{\mathrm{N}}$

$$
\left(\frac{\delta \epsilon_{N}}{\epsilon_{N}}\right)^{2}=\left(\frac{\delta \sigma_{X}}{\sigma_{X}}\right)^{2}+\left(\frac{\delta \sigma_{\theta}}{\sigma_{\theta}}\right)^{2}+\left(\frac{\delta p}{p}\right)^{2}
$$

can be expressed entirely in terms of the position resolution as

$$
\left(\frac{\delta \epsilon_{N}}{\epsilon_{N}}\right)^{2}=\left(\frac{\delta \sigma_{X}}{\sigma_{X}}\right)^{2}+\frac{2\left(\delta \sigma_{X}\right)^{2}}{D_{\theta}^{2} \sigma_{\theta}^{2}}+\frac{4\left(\delta \sigma_{X}\right)^{2}}{\alpha^{2} D_{D}^{2}}
$$

where $\mathrm{D}$ is the distance between chambers, and $\alpha$ is the dipole bend angle for the momentum measurement.

The expected fractional change in normalized emittance for the case described in Table 2 is about $23 \%$. However, in order to measure the smaller effects expected with lower initial emittances, we would like to determine $\Delta \varepsilon_{\mathrm{N}} / \varepsilon_{\mathrm{N}}$ to an accuracy of $0.1 \%$. Eq. 12 gives the accuracy required on the measurement of $\sigma_{\mathrm{x}}$. This can in turn be used to determine the chamber resolution required once the muon flux is known.

The position chamber sizes are determined by the beam divergence angles. The detectors for the beam incident on the experiment (P1-5) cover a position range of up to $\pm 3 \sigma$. In order to fully understand the performance of the cooling cell, we want to be sure that the chambers after the experiment can detect far into the tails of the angular distribution. For that reason these detectors (P6-10) cover a range of up to $\pm 4 \sigma$. Detectors P3-8 must consist of an $x$ and a y measuring plane. The other detectors only need to measure $\mathrm{x}$.

\section{Requirements for the AGS beam}

In order to measure individual tracks we propose using the slow extracted beam from the AGS. The useful muon rate should be larger than $\approx 10^{2} /$ spill so that a complete measurement can be made in about an hour, in order to minimize the effects of systematic errors. The useful rate should be smaller than $\approx 10^{4} /$ spill in order to use a fairly simple data acquisition system. The mean muon beam momentum at the experiment should be centered around $210 \mathrm{MeV} / \mathrm{c}$. A free region of about $6 \mathrm{~m}$ is required for the experiment following a hadron absorbermuon diffuser block. 
Table 3 summarizes the muon beam requirements.

\begin{tabular}{||l|l|l||}
\hline \multicolumn{2}{|c||}{ Table 3 } & Muon beam requirements \\
\hline $1 \sigma$ beam radius & 24 & $\mathrm{~cm}$ \\
\hline $1 \sigma$ beam divergence & 100 & $\mathrm{mrad}$ \\
\hline$\mu$ mean momentum & 211 & $\mathrm{MeV} / \mathrm{c}$ \\
\hline useful $\mu$ rate & $10^{2}-10^{4}$ & $\mu / \mathrm{spill}$ \\
\hline
\end{tabular}

The momentum following the experiments is measured with a dipole in the spectrometer area shown in Fig. 1. In order to guarantee that all tracks leaving the rod are measured, no quadrupoles are used in the spectrometer.

An appropriate incoming muon beam might be formed from pions produced by sending an incident proton beam into a target. This could be followed by a free space, pion decay region, which is sufficiently long to allow the muon beam to grow to the desired radius. Then the beam could enter a thick block of iron, for example, which would (1) allow the muon beam divergence to grow to the desired amount and (2) absorb out most of the remaining pions in the beam.

\section{Acknowledgements}

This research was supported by the U.S. Department of Energy under Contract No. DE-AC02-76CH00016. 


\section{References}

1. See, for example, Proc. of the Mini-workshop on $\mu^{+} \mu^{-}$colliders: particle physics and design, Napa, CA, Nuc. Instr. Meth. in Phys. Res. A350: 24-56, 1994.

2. See, for example, D.V. Neuffer, $\mu^{+} \mu^{-}$colliders: possibilities and challenges, Nuc. Instr. Meth. in Phys. Res. A350: 27-35, 1994; D. Neuffer, Principles and applications of muon cooling, Part. Accel. 14:75-90, 1983.

3. V.V. Parkhomchuk \& A.N. Skrinsky, Ionization cooling: physics and applications, Proc. 12th Int. Conf. on High Energy Accel., p. 485-7, 1983.

4. D.V. Neuffer \& R.B. Palmer, A high-energy high-luminosity $\mu^{+} \mu^{-}$collider, Proc. 1994 European Particle Accelerator Conf; R.B. Palmer, D.V. Neuffer \& J. Gallardo, A practical high-energy high-luminosity $\mathrm{p}^{+} \mu^{-}$collider, BNL report 61266. 